\title{
BMJ Open Cohort profile: the Buffalo OsteoPerio microbiome prospective cohort study
}

\author{
Hailey R Banack, ${ }^{1}$ Robert J Genco, ${ }^{2}$ Michael J LaMonte, ${ }^{1}$ Amy E Millen, ${ }^{1}$ \\ Michael J Buck, ${ }^{3}$ Yijun Sun, ${ }^{4}$ Christopher A Andrews, ${ }^{5}$ Kathleen M Hovey, ${ }^{1}$ \\ Maria Tsompana, ${ }^{6}$ Daniel I McSkimming, ${ }^{7}$ Jiwei Zhao, ${ }^{8}$ Jean Wactawski-Wende, ${ }^{1}$ \\ for the OsteoPerio Study Group
}

To cite: Banack HR, Genco RJ, LaMonte MJ, et al. Cohort profile: the Buffalo 0steoPerio microbiome prospective cohort study. BMJ Open 2018;8:e024263. doi:10.1136/ bmjopen-2018-024263

- Prepublication history for this paper is available online. To view these files, please visit the journal online (http://dx.doi. org/10.1136/bmjopen-2018024263).

Received 17 May 2018 Revised 29 August 2018 Accepted 15 October 2018

\section{Check for updates}

(C) Author(s) (or their employer(s)) 2018. Re-use permitted under CC BY-NC. No commercial re-use. See rights and permissions. Published by BMJ.

For numbered affiliations see end of article.

Correspondence to Dr Hailey R Banack; hrbanack@buffalo.edu

\section{ABSTRACT}

Purpose The Buffalo Osteoporosis and Periodontal Disease (0steoPerio) study is a prospective cohort study focused on the relationship between the microbiome and oral and systemic health outcomes in postmenopausal women. The cohort was established to examine how the oral microbiome is affected by (and how it affects) periodontal disease presence, severity and progression and to characterise the relationship between the microbiome, lifestyle habits and systemic disease outcomes.

Participants Participants $(n=1342)$ were postmenopausal women who were participating in the Women's Health Initiative observational study at the Buffalo, New York clinical centre. There were 1026 participants at the 5-year follow-up visit and 518 at the 15-year visit.

Findings to date Data collected include questionnaires, anthropometric measures, serum blood and saliva samples. At each clinic visit, participants completed a comprehensive oral examination to measure oral health and the oral microbiome. Preliminary findings have contributed to our understanding of risk factors for periodontal disease and the relationship between the oral microbiome and periodontal disease.

Future plans The novel microbiome data collected on a large sample of participants at three time points will be used to answer a variety of research questions focused on temporal changes in the microbiome and the relationship between the oral microbiome and oral and systemic disease outcomes. Little is currently known about the relationship between the oral microbiome and health outcomes in older adults; data from the 0steoPerio cohort will fill this gap. Microbiome samples are currently being analysed using next-generation sequencing technology with an anticipated completion date of late 2018.

\section{INTRODUCTION}

The Buffalo Osteoporosis and Periodontal Disease (OsteoPerio) study is a prospective cohort study that was established to explore risk factors for the development and progression of osteoporosis and periodontal disease in postmenopausal women. As technology has advanced, the focus of the cohort has evolved from traditional risk factor epidemiology to a novel exploration of the entire oral

\section{Strengths and limitations of this study}

- Unique, community-based cohort is a valuable resource to explore the relationship between the oral microbiome and systemic disease in older adults.

- Prospective study design and repeated clinic visits will allow us to examine change in the microbiome over time.

- Cohort will make a significant methodological contribution to the field of microbiome research and metagenomic analysis.

- As an ancillary study to the large Women's Health Initiative study, the participants only include postmenopausal women.

Most participants in the study had good oral hygiene.

microbiome and its relationship to health outcomes in postmenopausal women. This cohort will improve our understanding of the complex relationship between the oral microbiome and health and disease outcomes in older adults. It will also contribute to the growing body of literature focused on understanding the human microbiome. Up to this point, most studies of the microbiome have been descriptive reviews, cross-sectional or case-control studies rather than prospective studies with longitudinal follow-up. ${ }^{1}$

There has been a great deal of excitement about the potential of microbiome research in epidemiology and its application to clinical practice. ${ }^{1}$ Results from the Human Microbiome Project described the diversity, composition and function of the human microbiome, and recent calls have been made to further understand the role of the microbiome in disease aetiology. ${ }^{2}$ The microbiome is broadly defined as a collection of microbes living in and on our bodies, including bacteria, viruses and other organisms. ${ }^{1}$ Disruption of the microbiome is called dysbiosis, resulting in an imbalance between good and bad bacteria. Dysbiosis can result in an overabundance of bacteria, as is the case 
in periodontal disease, including the emergence of oral pathogens and reduction of commensal organisms. ${ }^{13}$ The concept of dysbiosis is central to understanding periodontal disease initiation and progression. ${ }^{45}$ Dysbiosis is thought to trigger immunoinflammatory processes that play a major role in the destruction of the soft tissues and bone supporting the teeth in the jaw. In a woman with osteoporosis, we would expect to see a greater reduction in oral bone and more severe periodontal disease, due to the combination of bacterial bone resorbing factors and lower local bone mineral density compared with a similarly aged woman without osteoporosis. ${ }^{6}$ There is scientific interest in understanding the factors that cause dysbiosis, as well as understanding the factors that restore a dysbiotic microbiome to a healthy state. ${ }^{1}$

Technological advances have recently enabled the exploration of the human microbiome in large epidemiological cohorts. ${ }^{7-9}$ The samples collected from the OsteoPerio cohort will be analysed using innovative next-generation sequencing technology, including high-throughput DNA sequencing. ${ }^{7}$ Our objectives are to provide new information on the composition and diversity of the oral microbiome in older women; to examine how the oral microbiome is affected by (and how it affects) periodontal disease presence, severity and progression; and characterise the relationship between the microbiome and lifestyle habits and other factors (ie, smoking, diabetes, diet). Results from this cohort study will make substantive contributions to the field of microbiome research as well as methodological contributions by developing and implementing rigorous quality control procedures and statistical methods for analysing large metagenomic data.

\section{COHORT DESCRIPTION}

\section{Study population and setting}

The participants of the OsteoPerio cohort were recruited from participants in the Women's Health Initiative (WHI) observational study (WHI-OS) at the Buffalo, New York clinical centre. ${ }^{10}$ The study was approved by the Institutional Review Board at the University at Buffalo. The OsteoPerio cohort is an ancillary study to the WHI, a major US research study of postmenopausal women, aimed at preventing morbidity and mortality in postmenopausal women. ${ }^{11-13}$ WHI participants from the Buffalo centre were recruited from 1993 to 1998 . The OsteoPerio cohort was recruited approximately 3 years after WHI enrolment between 1997 and 2001. Study visits took place at baseline (1997-2001; $\mathrm{n}=1342)$, 5 years $(2002-2005 ; \mathrm{n}=1026)$ and 15 years $(2014-2018 ; n=518)$. Figure 1 describes the flow of recruitment and retention of OsteoPerio participants. Table 1 describes demographic and anthropometric characteristics of the study cohort, stratified by visit (baseline, 5 years, 15 years) .

Women who were enrolled in the WHI-OS who had fewer than six teeth, bilateral hip replacement, history of bone disease other than osteoporosis, history of cancer within previous 10 years or treatment for serious illness were ineligible for enrolment into the OsteoPerio study. ${ }^{14}$ At the 5-year follow-up, women were ineligible to participate if they had developed cancer or other immunosuppressive disorders, had cirrhosis or chronic hepatitis $\mathrm{B}$ or bilateral hip replacement in the interim since their baseline study visit. Additionally, there was a temporary restriction on women who had recently taken antibiotics, had recent diagnostic imaging involving contrast dye or had a dental cleaning in the past 30 days; they were ineligible to participate for the 30-day period after antibiotics / imaging/dental visit but eligible thereafter. Women who had exposure to dental X-rays within the year prior to study visit were temporarily ineligible until at least a year had passed since their last dental X-ray. ${ }^{14}$ At the $15^{+}$-year follow-up, the criteria remained the same with a slight modification for women who had been diagnosed with cancer: women currently undergoing treatment for invasive cancer were ineligible, but women were eligible to participate if 6 months had elapsed since their last cancer treatment.

\section{Data collection and measures Overview}

Participants were scheduled for morning clinic appointments to allow for fasting blood measurements at each of their study visits. On the day of the visit, participants completed an informed consent form and study questionnaires. Blood sample and saliva collection, blood pressure measurements and anthropometric measurements were then completed. A comprehensive whole-mouth oral examination was performed by a dental examiner. Each component of the clinic visit is detailed below, and was repeated at baseline, year 5 , and year 15 .

\section{Oral examination}

Subgingival samples were taken from 12 index teeth or their substitutes using a paper point technique. ${ }^{615}$ Plaque was assessed at three surfaces per tooth. The paper point technique involved drying each tooth with cotton (to reduce cross contamination), placing absorbent paper points (Johnson \& Johnson) into the gingival pocket of the mesiobuccal surface of the selected tooth for $10 \mathrm{~s}$, transferring the paper point to $4 \mathrm{~mL}$ of Ringer's solution, vortexing the samples to disperse the organisms, transferring $0.5 \mathrm{~mL}$ to cryogenic straws at baseline and 5 years and storing in liquid nitrogen in $-80^{\circ} \mathrm{C}$ freezers. At the 15 -year study, samples were transferred into cryovials and frozen at $-80^{\circ} \mathrm{C}$ directly after collection. ${ }^{6}$ Upper and lower arches from each of the three time points were pooled and transferred to cryovials for microbiome testing. Participants also provided $5 \mathrm{~mL}$ of saliva, collected in a conical tube, over a $10 \mathrm{~min}$ period. Cytokines and $\mathrm{C}$ reactive protein were measured in saliva. ${ }^{16}$

The oral examination included examination of the head and neck, oral mucous membranes, recording of restorative appliances, as well as caries and missing teeth. Gingival assessment was done on three gingival sites per 


\section{2,249 Buffalo WHIOS}

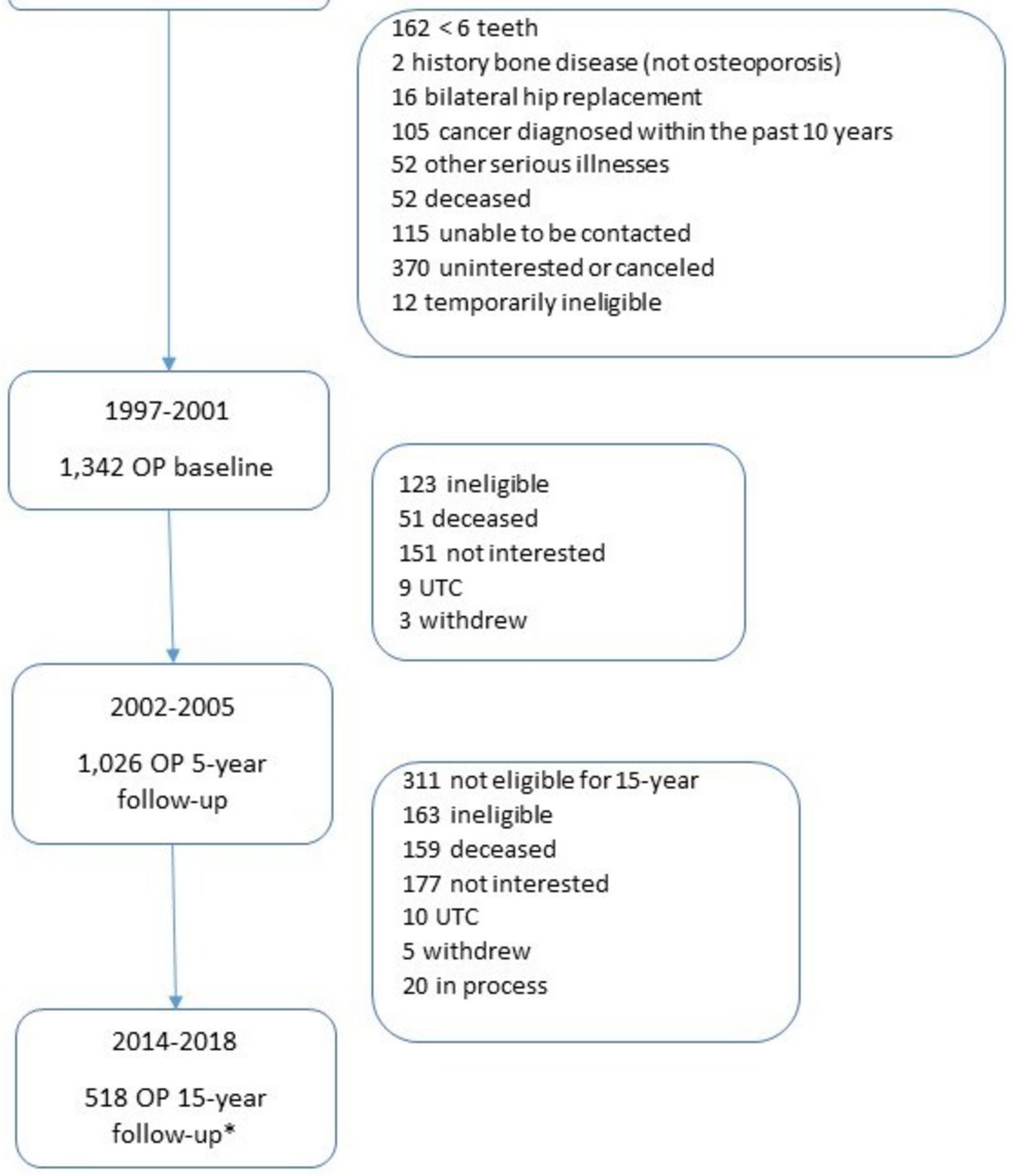

Figure 1 Flow chart detailing participant enrolment and losses to follow-up in the Osteoporosis and Periodontal (OP) Disease study. *As of Jan 2018; temporary ineligibility was due to a variety of different reasons (ie, recent oral X-rays or radio luminescent dye tests). OS, observational study; WHI, Women's Health Initiative; UTC, unable to contact.

tooth of all teeth present by scoring bleeding on probing. Calculus presence was assessed for each tooth on a threepoint scale. Pocket depth, the distance in millimetres from the gingival margin of the tooth to the base of the sulcus/ pocket, was measured on six surfaces per tooth of all the teeth present except the third molars using a constant force electronic periodontal probe (The Florida Probe System, Gainsville, FL, USA). ${ }^{17}$ The final component of the oral examination was oral radiographs (X-rays). Up to 11 standardised intraoral radiographs were taken from each participant and digitised; the number of radiographs taken depended on the number of teeth present. ${ }^{6}$

\section{Blood measures}

Fasting blood samples were drawn by trained technicians using venipuncture, and then centrifuged, portioned to 
Table 1 Characteristics of study participants at baseline, 5-year and 15-year follow-up visits

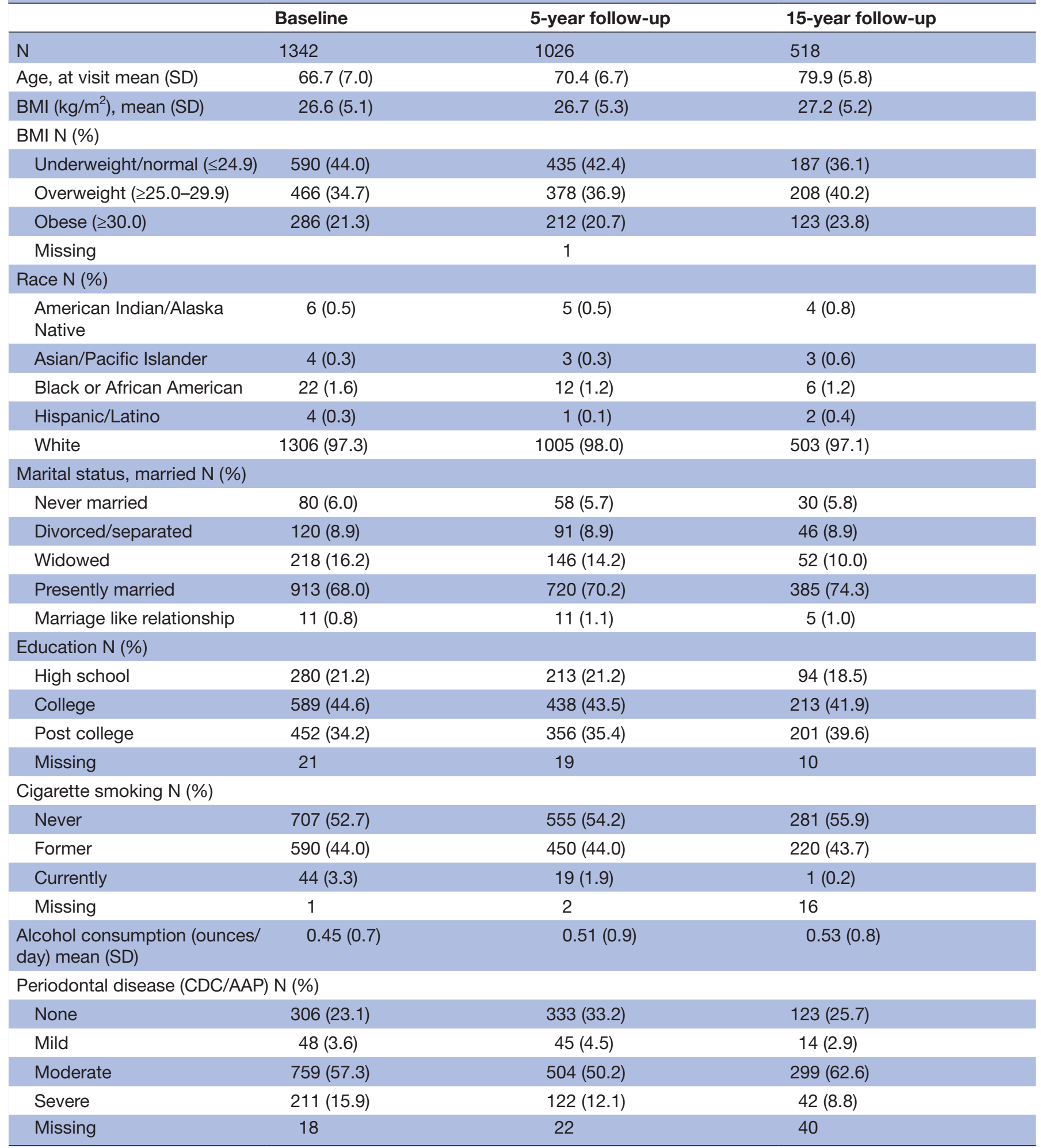

AAP, American Academy of Periodontology; BMI, body mass index; CDC, Centers for Disease Control and Prevention.

serum, plasma and buffy coat, and frozen either in liquid nitrogen (baseline, year 5 ) or in $-80^{\circ} \mathrm{C}$ freezers (year 15) in $0.5 \mathrm{~mL}$ aloquots. All samples are housed in the University at Buffalo's Department of Epidemiology and Environmental Health biospecimen bank. ${ }^{18}$ Blood samples have been used to measure serum lipids, C reactive protein, blood glucose, blood insulin, comprehensive metabolic panel, reproductive hormones, 25-hydroxyvitamin D and several cytokines. Additional sample remains for future use in the biospecimen bank. An overview of laboratory variables already measured at each study visit is presented in table 2 . 
Table 2 Comprehensive list of analytes collected at each Osteoporosis and Periodontal Disease study visit listed alphabetically

\begin{tabular}{|c|c|c|}
\hline $\begin{array}{l}\text { Alanine aminotransferase (SGPT), } \\
\text { unit/L }\end{array}$ & LDL cholesterol (direct), mg/dL & Serum insulin $(\mathrm{pg} / \mathrm{mL})$ \\
\hline Albumin, g/dL & Plasma 25(OH)D (nM) & Serum leptin (pg/mL) \\
\hline Alkaline phosphatase, unit/L & Potassium, mmol/L & Serum MCP-1 $(\mathrm{pg} / \mathrm{mL})$ \\
\hline $\begin{array}{l}\text { Aspartate aminotransferase (SGOT), } \\
\text { unit/L }\end{array}$ & Protein, g/dL & Serum MMP-2 (pg/mL) \\
\hline Bilirubin, mg/dL & Saliva adiponectin $(\mathrm{pg} / \mathrm{mL})$ & Serum MMP-8 (pg/mL) \\
\hline Blood urea nitrogen, $\mathrm{mg} / \mathrm{dL}$ & Saliva CRP (pg/mL) & Serum MMP-9 (pg/mL) \\
\hline Calcium, mg/dL & Saliva insulin $(p g / m L)$ & Serum OPG (pg/mL) \\
\hline Carbon dioxide, $\mathrm{mmol} / \mathrm{L}$ & Saliva MCP-1 (pg/mL) & Serum PTH $(\mathrm{pg} / \mathrm{mL})$ \\
\hline Chloride, $\mathrm{mmol} / \mathrm{L}$ & Saliva MMP-8 (pg/mL) & Serum TNF- $\alpha(p g / m L)$ \\
\hline Cholesterol/HDL ratio & Saliva OPG (pg/mL) & SHBG (nmol/L) \\
\hline Creatinine, mg/dL & Saliva TNF- $\alpha$ (pg/mL) & Sodium, mmol/L \\
\hline DHEA-S (ug/dL) & Serum adiponectin $(\mathrm{pg} / \mathrm{mL})$ & Testosterone (ng/mL) \\
\hline Estradiol $(p g / m L)$ & Serum CRP (pg/mL) & Total cholesterol, mg/dL \\
\hline Glomerular filtration rate, $\mathrm{mL} / \mathrm{min} / 1.73 \mathrm{~m}^{2}$ & Serum IL-10 (pg/mL) & Triglycerides, mg/dL \\
\hline Glucose, mg/dL & Serum IL-2 (pg/mL) & VLDL cholesterol (calculated), mg/dL \\
\hline HDL cholesterol, mg/dL & Serum IL-4 (pg/mL) & \\
\hline Insulin (uU/mL) & Serum IL-6 (pg/mL) & \\
\hline LDL cholesterol (calculated), mg/dL & Serum IL-8 (pg/mL) & \\
\hline
\end{tabular}

CRP, C reactive protein; SGPT,serum glutamic pyruvic transaminase; LDL, low density lipoprotein; MCP , monocyte chemotactic protein; SGOT, serum glutamic oxaloacetic transaminase; MMP,matrix metalloproteinase; OPG, osteoprotegerin; PTH, parathyroid hormone; TNF, tumor necrosis factor; HDL, high density lipoprotein; DHEA-S, dehydroepiandrosterone-sulfate; VLDL, very low density lipoprotein.

\section{Study questionnaires}

Demographic information and personal characteristics were assessed via self-report questionnaires. Questionnaires collected information on demographic factors, medical and oral health history, dental hygiene, smoking, dietary intake (via Food Frequency Questionnaire), medications and dietary supplements and use of menopausal hormone therapy. ${ }^{11} 19$ As part of the larger WHI study, participants were asked questions about a wide range of medical, psychological and behavioural factors, health conditions and diseases. Annual assessments to update health outcomes are part of the WHI study. ${ }^{20}$

\section{Anthropometric measures}

All measures were conducted by trained and certified staff according to standardised protocols. Height and weight were measured by a trained technician using a calibrated wall-fixed clinical stadiometer and weight scale. Waist circumference was measured at the narrowest part of the torso at end exhalation using an anthropometric tape measure. Systolic and diastolic blood pressure were measured after 5 min of quiet sitting with legs uncrossed using a manual sphygmomanometer. ${ }^{18}$ Body composition, including fat mass, lean mass and both total-body and site-specific bone mineral density, was assessed using dual energy X-ray absorptiometry (Hologic QDR-4500). ${ }^{17}$

\section{Periodontal disease measures}

Periodontal disease presence and severity were defined using two definitions, one derived from the OsteoPerio study based on alveolar crestal height (ACH), a measure of oral bone loss, and tooth loss ${ }^{10}$ and the other from the Centers for Disease Control and Prevention/American Academy of Periodontology (CDC/AAP). ${ }^{21}$ The OsteoPerio definition categorised participants into none (wholemouth mean $\mathrm{ACH}<2 \mathrm{~mm}$, no tooth loss due to periodontal disease), mild/moderate (whole-mouth mean ACH $\geq 2$ and $<3 \mathrm{~mm}$ or one or more sites with $\geq 4 \mathrm{~mm}$ and no tooth loss due to periodontal disease) and severe (whole-mouth mean $\mathrm{ACH} \geq 3 \mathrm{~mm}$ or more than two sites with $\geq 5 \mathrm{~mm}$ or $\geq 1$ tooth loss due to periodontal disease). ${ }^{14}$ The CDC/ AAP definition used none/mild (neither moderate nor severe disease), moderate ( $\geq 2$ interproximal sites with clinical attachment loss (CAL) $\geq 4 \mathrm{~mm}$, not on the same tooth, or $\geq 2$ interproximal sites with CAL $5 \mathrm{~mm}$, not on the same tooth) and severe ( $\geq 2$ interproximal sites with $\mathrm{CAL} \geq 6 \mathrm{~mm}$, not on the same tooth, and $\geq 1$ interproximal site with pocket depth $\geq 5 \mathrm{~mm}) .{ }^{21}$ The actual measured values for each of the measures obtained are also available for each participant by tooth and as whole-mouth mean and worst site values.

\section{Analysis of the oral microbiome}

The composition and diversity of the oral subgingival microbiome were assessed by $16 \mathrm{~S}$ ribosomal DNA 
(rDNA) sequencing with the Illumina high-throughput MiSeq platform. ${ }^{22}$ Bacterial DNA was isolated from participant subgingival plaque samples and put on a PCR plate. Sequencing libraries were prepared using a two-step PCR method for targeting the V3-V4 region (464 bp) of the $16 \mathrm{~S}$ rDNA. The first PCR (25-cycle) used V3 (341F) forward and V4 (805R) reverse primer pairs with added Illumina adapter overhang nucleotide sequences. The first round PCR product was purified and Nextera XT Indexes were added with eight PCR cycles. Unique pairs of indexes were added to each sample allowing the pooling of all 96 samples in a sequencing lane. Sample quality was assessed by both DNA concentration and capillary electrophoresis. Samples were then normalised and pooled (96 per run). The final concentration of the pooled sequence library was then determined by qPCR and diluted to a final concentration of $12 \mathrm{pM}$ with $20 \%$ PhiX. Sequencing was performed using the Illumina MiSeq system $2 \times 300$ base paired-end sequencing method. ${ }^{22}$

Quality control was systematically evaluated throughout the sample preparation and sequencing procedures. Each PCR plate has 96 wells and contained 88 study samples, as well as positive and negative control samples (three plaque pools, one mock DNA, three extraction buffers, and one DNA-free water). The plaque pool samples were generated by pooling subgingival plaque samples together from several participants to create a single homogenous sample that was portioned out into vials and frozen. These standard, blinded quality control samples served as a positive control for the entire process allowing for the evaluation of variability in DNA extraction, PCR amplification and sequencing. The mock DNA sample was added after DNA extraction and represents a positive control for PCR amplification and sequencing. The extraction buffer sample was a negative control treated at all steps of DNA extraction, PCR amplification and sequencing. This negative control allowed us to detect contamination from the DNA extraction step. The DNA-free water is a negative control at the PCR stage and allowed us to detect contamination during PCR amplification.

\section{Findings to date}

Traditional risk factors for periodontal disease

Results from the OsteoPerio study have supported the link between bone mineral density and periodontal disease. Compared with women with normal systemic bone mineral density T-score, women with osteoporosis are nearly twice as likely to have oral bone loss, measured by $\mathrm{ACH}(\mathrm{OR}=1.90 ; 95 \%$ CI 1.19 to 3.05$) .{ }^{10}$ The relationship between systemic bone mineral density and oral bone loss is greater in women over age $70(\mathrm{OR}=3.57 ; 95 \%$ CI 1.42 to 8.97) compared with women less than age $70(\mathrm{OR}=1.63$; $95 \%$ CI 2.83). ${ }^{10}$ CAL, a measure of periodontal disease severity, was inversely associated with bone mineral density in the OsteoPerio study. ${ }^{17}$ Other results from our cohort have demonstrated that cigarette smoking and indicators of periodontal disease severity are strong risk factors for incident tooth loss in older women. ${ }^{23}{ }^{24}$ Examination of longitudinal measures of periodontal disease over the first 5 years of follow-up among postmenopausal women in the OsteoPerio study revealed that, as expected, the prevalence and severity of periodontal disease increased as women age. ${ }^{14}$ Additional published study findings have describe the relationship of clinically measured periodontal disease with tooth loss, ${ }^{23-25}$ metabolic syndrome, ${ }^{18}$ plasma 25-hydroxyvitamin $\mathrm{D}$ concentrations, ${ }^{525-27}$ endogenous serum oestrogen concentrations ${ }^{4}$ and incident cancer, ${ }^{28}$ as well as the association between the presence of subgingival periodontal pathogens and incident cancer. ${ }^{29}$

We found a strong positive association between specific oral bacteria and severe oral bone loss, an indicator of periodontal disease. ${ }^{630}$ Infection with Porphyromonas gingivalis ( $\mathrm{OR}=2.34 ; 95 \%$ CI 1.66 to 3.29), Tannerella forsythia $(\mathrm{OR}=1.76 ; 95 \%$ CI 1.34 to 2.30$)$, Prevotella intermedia (1.40; 95\% CI 1.07 to 1.83 ) and Campylobacter rectus $(\mathrm{OR}=1.85$; 95\% CI 1.33 to 2.57 ) were associated with an increased likelihood of oral bone loss, controlling for age, smoking status and income. ${ }^{6}$

\section{Oral microbiome}

There were differences in the microbial composition and diversity among OsteoPerio participants with and without periodontal disease (figure 2). Among 30 women included in a pilot analysis, 15 (figure 2; labelled N1-15 on x-axis) with no periodontal disease and 15 (N16-30) with disease, there were noticeable differences in the relative abundance of commensal and pathogenic periodontal bacteria. Women with no periodontitis (N1-15) have a greater relative abundance of organisms associated with periodontal health (represented by blue, green and yellow colours), whereas women with periodontal disease (N16-30) have a greater abundance of potential pathogenic microorganisms (represented by red and orange). The colours in the figure correspond with the Socransky classification for oral bacteria. ${ }^{31}$ Figure 2 also demonstrates the variation in the microbiome composition among women with the same periodontal status, for example, participants 1,8 and 11 have no periodontal disease but were found to have pathogenic bacteria (red/orange bacteria), while participants 19, 21 and 26 have severe periodontal disease but an abundance of non-pathogenic bacteria (blue/yellow/green).

\section{Strengths and limitations}

The OsteoPerio cohort fills an important gap in our understanding of the relationship between the oral microbiome and disease outcomes. The prospective study design, cohort size and linkage with larger WHI study are key strengths. To the best of our knowledge, the OsteoPerio study is the first study able to answer questions about how the oral microbiome changes as women age, determine the relationship of the oral microbiome composition and periodontal disease initiation and progression, and explore the association of the oral microbiome with diseases relevant to ageing. The use of 


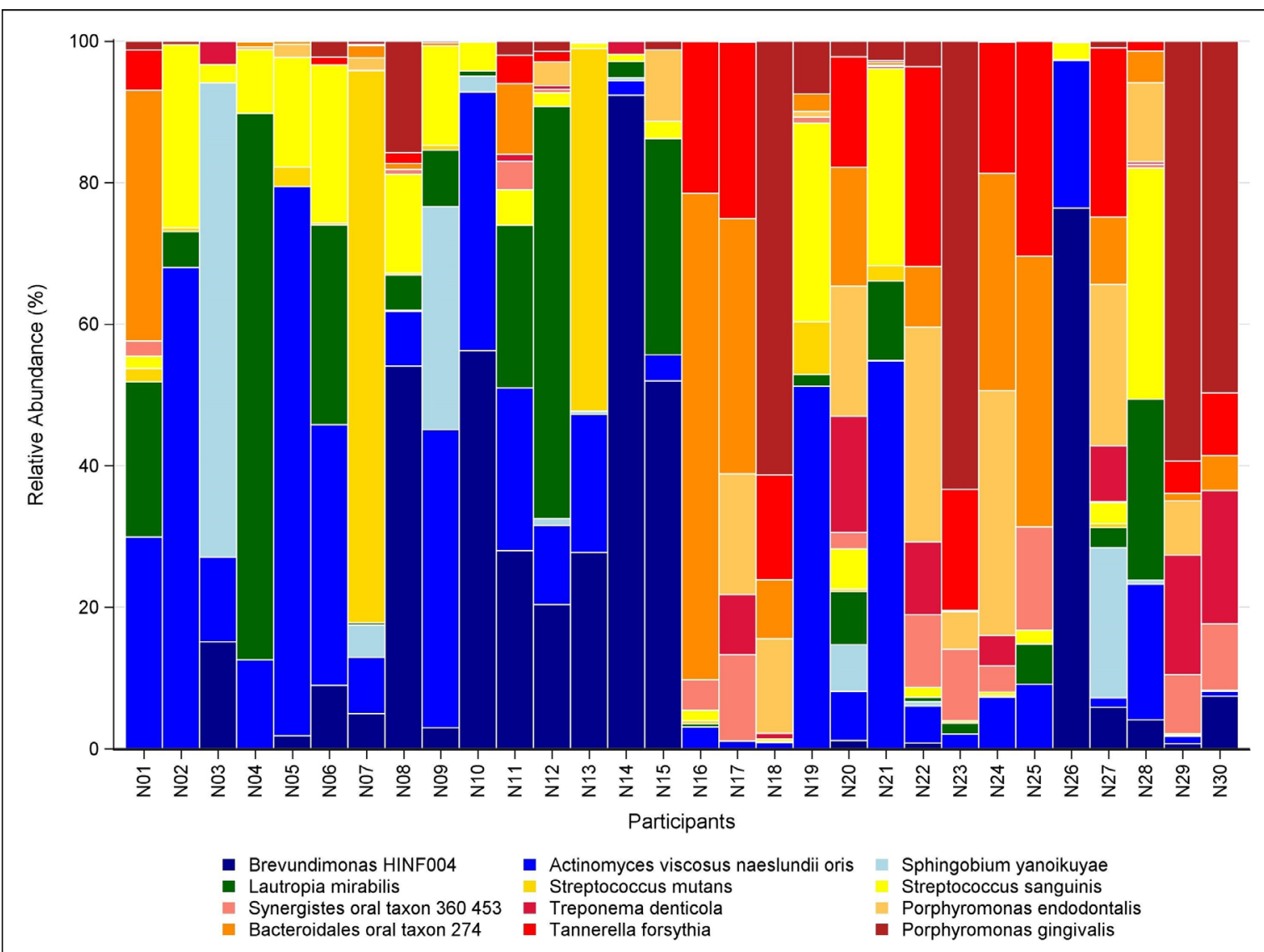

Figure 2 Microbiome composition and diversity of pilot sample of 30 OsteoPerio participants (N1-N30; X-axis). The figure demonstrates the relative abundance (y-axis) of 12 selected bacteria according to their known association with health and disease. Bacteria known to be associated with oral health are represented by blue, green and yellow colours. Bacteria known to be associated with oral disease are represented by red and orange colours.

novel metagenomics technology is another strength as the data generated from this cohort lie at the intersection of epidemiology, microbiology, bioinformatics and biostatistics. Once $16 \mathrm{~S}$ rDNA sequencing is complete, the data can be used to answer an extremely wide range of questions about the oral microbiome and disease outcomes. The main limitation of the cohort is that the participants are all postmenopausal women, who are predominantly white and well educated. Additionally, participants were required to have at least six teeth to participate in the study and most women had good oral hygiene. This may affect the study findings because those with very severe periodontal disease may have had teeth extracted (resulting in fewer than six teeth present in the mouth) and individuals with good oral hygiene may have a lower oral bacterial load than those who have poor oral hygiene.

We also learned some valuable lessons applicable to researchers interested in microbiome research. Most valuable, perhaps, is the importance of collaborating with investigators across disciplines when assembling cohort studies for human microbiome research. Collaboration between researchers with diverse areas of expertise (epidemiology, dentistry/oral biology, statistics, biochemistry and bioinformatics) has enabled this study to be successful. Another lesson learned is the high degree of technical knowledge required to conduct microbiome analyses, including developing standard operating procedures, quality control metrics, computational strategies and choosing reference databases. It took a very long time to develop and implement these procedures because the field of microbiome research is still an emerging discipline and there are few 'best practice' guidelines to follow.

\section{Patient and public involvement}

OsteoPerio participants were given the opportunity to provide feedback on study visits (ie, visits too short, too long). As well, after the baseline visit, we conducted a survey to ask study participants what they used the study results for; whether they shared the results with their physician or made any lifestyle changes. Study results and updates are continually shared with OsteoPerio participants through mailed newsletters and in-person meetings. The purpose of the in-person meetings is twofold: to disseminate study findings and provide participants with the opportunity to ask about their research interests and priorities.

\section{Author affiliations}

${ }^{1}$ Department of Epidemiology and Environmental Health, University at Buffalo, The State University of New York, Buffalo, New York, USA

${ }^{2}$ Departments of Oral Biology, and Microbiology and Immunology, and Center for Microbiome Research, University at Buffalo, The State University of New York, Buffalo, New York, USA 
${ }^{3}$ Departments of Biochemistry and Bioinformatics, University at Buffalo, The State University of New York, Buffalo, New York, USA

${ }^{4}$ Department of Computer Science and Engineering, University at Buffalo, The State University of New York, Buffalo, New York, USA

${ }^{5}$ Department of Ophthalmology and Visual Sciences, University of Michigan, Ann Arbor, Michigan, USA

${ }^{6}$ Center of Excellence in Bioinformatics and Life Sciences, University at Buffalo, The State University of New York, Buffalo, New York, USA

${ }^{7}$ Genome, Environment and Microbiome Community of Excellence, University at Buffalo, The State University of New York, Buffalo, New York, USA

${ }^{8}$ Department of Biostatistics, University at Buffalo, The State University of New York, Buffalo, New York, USA

Acknowledgements Program Office: (National Heart, Lung, and Blood Institute, Bethesda, Maryland) Jacques Rossouw, Shari Ludlam, Joan McGowan, Leslie Ford, and Nancy Geller Clinical Coordinating Center: (Fred Hutchinson Cancer Research Center, Seattle, WA) Garnet Anderson, Ross Prentice, Andrea LaCroix, and Charles Kooperberg Investigators and Academic Centers: (Brigham and Women's Hospital, Harvard Medical School, Boston, MA) JoAnn E Manson; (MedStar Health Research Institute/Howard University, Washington, DC) Barbara V. Howard; (Stanford Prevention Research Center, Stanford, CA) Marcia L Stefanick; (The Ohio State University, Columbus, OH) Rebecca Jackson; (University of Arizona, Tucson/Phoenix, AZ) Cynthia A Thomson; (University at Buffalo, Buffalo, NY) Jean Wactawski-Wende; (University of Florida, Gainesville/Jacksonville, FL) Marian Limacher; (University of lowa, lowa City/Davenport, IA) Jennifer Robinson; (University of Pittsburgh, Pittsburgh, PA) Lewis Kuller; (Wake Forest University School of Medicine, WinstonSalem, NC) Sally Shumaker; (University of Nevada, Reno, NV) Robert Brunner; (University of Minnesota, Minneapolis, MN) Karen L Margolis Women's Health Initiative Memory Study: (Wake Forest University School of Medicine, WinstonSalem, NC) Mark Espeland.

\section{Collaborators OsteoPerio Study Group.}

Contributors JW-W, RG, MJL, AM, MB, YS, CAA and KMH were responsible for the study design, planning and data collection. MT and MB were responsible for developing the protocol for analysing microbiome samples. DM, YS and $\mathrm{JZ}$ were responsible for bioinformatics and analysis. HRB was responsible for writing the initial draft of the manuscript, and subsequent drafts were reviewed by all authors listed. All authors had input on interpretation and reporting of study findings. All authors provided approval for the published version of this manuscript.

Funding The Women's Health Initiative program is funded by the National Heart, Lung, and Blood Institute, National Institutes of Health (NIH), U.S. Department of Health and Human Services through contracts HHSN268201600018C, HHSN268201600001C, HHSN268201600002C, HHSN268201600003C, and HHSN268201600004C; JW-W: R01DE013505 from the National Institute of Dental and Craniofacial Research, NIH, U.S. Army, Medical Research and Materiel Command, grant OS950077 and $\mathrm{NIH/National} \mathrm{Heart} \mathrm{Lung} \mathrm{and} \mathrm{Blood} \mathrm{Institute}$ contract N01WH32122; YS and RG, JW-W: R01Al125982; HRB: Canadian Institute of Health Research Banting Postdoctoral Fellowships Program.

Competing interests None declared.

Patient consent Not required.

Ethics approval State University of New York at Buffalo IRB.

Provenance and peer review Not commissioned; externally peer reviewed.

Data sharing statement We are actively interested in collaborating with investigators with shared interests in understanding the links between the oral microbiome and systemic disease. Data on the oral microbiome, blood and urine specimens, clinical and anthropometric measures, and participant characteristics are all potentially available for collaborative analyses. Investigators interested in using the 0steoPerio study data and collaborating with the study team are encouraged to contact the principal investigator, Dr. Jean Wactawski-Wende at jww@buffalo.edu.

Open access This is an open access article distributed in accordance with the Creative Commons Attribution Non Commercial (CC BY-NC 4.0) license, which permits others to distribute, remix, adapt, build upon this work non-commercially, and license their derivative works on different terms, provided the original work is properly cited, appropriate credit is given, any changes made indicated, and the use is non-commercial. See: http:// creativecommons.org/licenses/by-nc/4.0/.

\section{REFERENCES}

1. Foxman B, Martin ET. Use of the microbiome in the practice of epidemiology: A primer on -omic technologies. Am J Epidemiol 2015;182:1-8.

2. Foxman B, Rosenthal M. Implications of the human microbiome project for epidemiology. Am J Epidemiol 2013;177:197-201.

3. Lane ER, Zisman TL, Suskind DL. The microbiota in inflammatory bowel disease: current and therapeutic insights. J Inflamm Res 2017;10:63-73.

4. Wang $\mathrm{Y}$, LaMonte MJ, Hovey KM, et al. Association of serum $17 \beta$-estradiol concentration, hormone therapy, and alveolar crest height in postmenopausal women. J Periodontol 2015;86:595-605.

5. Sahli MW, Wactawski-Wende J, Ram PK, et al. Association of plasma 25-hydroxyvitamin d concentrations and pathogenic oral bacteria in postmenopausal females. J Periodontol 2014;85:944-55.

6. Brennan RM, Genco RJ, Wilding GE, et al. Bacterial species in subgingival plaque and oral bone loss in postmenopausal women. $J$ Periodontol 2007;78:1051-61.

7. Demmer RT. Invited commentary: The microbiome and population health-considerations for enhancing study design and data analysis in observational and interventional epidemiology. Am J Epidemiol 2018;187:1291-4.

8. Project; HM. Structure, function and diversity of the healthy human microbiome. Nature 2012;486:207-14.

9. Sinha R, Goedert JJ, Vogtmann E, et al. Quantification of human microbiome stability over 6 months: Implications for epidemiologic studies. Am J Epidemiol 2018;187:1282-90.

10. Wactawski-Wende J, Hausmann E, Hovey K, et al. The Association between osteoporosis and alveolar crestal height in postmenopausal women. J Periodontol 2005;76 Suppl 11S:2116-24.

11. Langer RD, White E, Lewis CE, et al. The women's health initiative observational study: baseline characteristics of participants and reliability of baseline measures. Ann Epidemiol 2003;13-S107-S121.

12. Rossouw J, Anderson G, Prentice R, et al. Risks and benefits of estrogen plus progestin in healthy postmenopausal women: Principa results from the women's health initiative randomized controlled trial. JAMA 2002;288:321-33.

13. The Women's Health Initiative Study G. Design of the Women's Health Initiative Clinical Trial and Observational Study. Controlled Clinical Trials 1998;19:61-109.

14. LaMonte MJ, Hovey KM, Genco RJ, et al. Five-year changes in periodontal disease measures among postmenopausal females: the Buffalo OsteoPerio study. J Periodontol 2013;84:572-84.

15. Rosenberg DB. The paper point technique: Part 1. Dentistry Today 2003;22:80-6.

16. Browne RW, Kantarci A, LaMonte MJ, et al. Performance of multiplex cytokine assays in serum and saliva among community-dwelling postmenopausal women. PLoS One 2013;8:e59498.

17. Brennan RM, Genco RJ, Hovey KM, et al. Systemic bone density, and subgingival calculus in postmenopausal women. J Periodonto 2007;78:2104-11.

18. LaMonte MJ, Williams AM, Genco RJ, et al. Association between metabolic syndrome and periodontal disease measures in postmenopausal women: the Buffalo OsteoPerio study. J Periodontol 2014;85:1489-501.

19. Patterson RE, Kristal AR, Tinker LF, et al. Measurement characteristics of the Women's Health Initiative food frequency questionnaire. Ann Epidemiol 1999;9:178-87.

20. Design of the women's health initiative clinical trial and observational study. Control Clin Trials 1998;19:61-109.

21. Rc P, Eke P. Case definitions for use in population-based surveillance of periodontitis. Journal of Periodontology 2007;78:1387-99.

22. Zheng W, Tsompana M, Ruscitto A, et al. An accurate and efficient experimental approach for characterization of the complex oral microbiota. Microbiome 2015;3:48.

23. Bole C, Wactawski-Wende J, Hovey KM, et al. Clinical and community risk models of incident tooth loss in postmenopausal women from the Buffalo Osteo Perio Study. Community Dent Oral Epidemiol 2010;38:487-97.

24. Mai X, Wactawski-Wende J, Hovey KM, et al. Associations between smoking and tooth loss according to the reason for tooth loss. J Am Dent Assoc 2013;144:252-65.

25. Pavlesen S, Mai X, Wactawski-Wende J, et al. Vitamin D Status and tooth loss in postmenopausal females: The buffalo osteoporosis and periodontal disease (OsteOperio) study. J Periodontol 2016;87:852-63.

26. Millen AE, Andrews CA, LaMonte MJ, et al. Vitamin D status and 5-year changes in periodontal disease measures among 
postmenopausal women: the Buffalo OsteoPerio Study. $J$ Periodontol 2014;85:1321-32.

27. Millen AE, Hovey KM, LaMonte MJ, et al. Plasma 25-hydroxyvitamin $\mathrm{D}$ concentrations and periodontal disease in postmenopausal women. J Periodontol 2013;84:1243-56.

28. Mai X, LaMonte MJ, Hovey KM, et al. Periodontal disease severity and cancer risk in postmenopausal women: the Buffalo OsteoPerio Study. Cancer Causes Control 2016;27:217-28.
29. Mai X, Genco RJ, LaMonte MJ, et al. Periodontal pathogens and risk of incident cancer in postmenopausal females: The buffalo osteoperio study. J Periodontol 2016;87:257-67.

30. Brennan-Calanan RM, Genco RJ, Wilding GE, et al. Osteoporosis and oral infection: independent risk factors for oral bone loss. J Dent Res 2008;87:323-7.

31. Socransky SS, Haffajee AD, Cugini MA, et al. Microbial complexes in subgingival plaque. J Clin Periodontol 1998;25:134-44. 